\title{
Telling the Truth to Child Cancer Patients in COVID-19 Times
}

\author{
Lynn Gillam $($ Merle Spriggs • Clare Delany • \\ Rachael Conyers • Maria McCarthy
}

Received: 12 May 2020 / Accepted: 21 September 2020

(C) Journal of Bioethical Inquiry Pty Ltd. 2020

\begin{abstract}
A notable feature of the COVID-19 pandemic is that children are less at risk of becoming infected or, if infected, less likely to become seriously unwell, so ethical discussions have consequently focused on the adult healthcare setting. However, despite a lower risk of children becoming acutely ill with COVID-19, there nevertheless may be significant and potentially
\end{abstract}

L. Gillam . M. Spriggs : C. Delany

Children's Bioethics Centre, Royal Children's Hospital,

Melbourne, Australia

e-mail: mspriggs@unimelb.edu.au

e-mail: merle.spriggs@mcri.edu.au

e-mail: c.delany@unimelb.edu.au

L. Gillam $(\bowtie)$ : M. Spriggs

Melbourne School of Population and Global Health,

The University ofMelbourne, 50 Flemington Road, Parkville, Victoria 3052, Australia

e-mail: Lynn.gillam@rch.org.au

e-mail: 1.gillam@unimelb.edu.au

\section{Spriggs}

Honorary Research Fellow, Murdoch Children's Research

Institute, Melbourne, Australia

\section{Delany}

Department of Medical Education, Melbourne Medical School, The University of Melbourne, 50 Flemington Road, Parkville, Victoria 3052, Australia

C. Delany

Peter MacCallum, Cancer Centre, Melbourne, Australia sustained effects of COVID-19 on the physical, psychological, and emotional health and well-being of children. Focusing on the context of children's cancer care, and specifically bone marrow transplant (BMT), we describe some of these effects and then address one specific ethical challenge that arises. That is the question of what and how much to tell children whose cancer

\section{R. Conyers}

Children's Cancer Centre, The Royal Children's Hospital, Melbourne, Australia

e-mail: Rachel.conyers@ rch.org.au

R. Conyers : M. McCarthy

Department of Paediatrics, University of Melbourne,

50 Flemington Road, Parkville, Victoria 3052, Australia

M. McCarthy

e-mail: maria.mccarthy@rch.org.au

R. Conyers

Cardiac Regeneration, Cell Biology, Murdoch Children's

Research Institute, Melbourne, Australia

M. McCarthy

Psycho-oncology Program, The Royal Children's Hospital, Melbourne, Australia

M. McCarthy

Brain and Mind, Murdoch Children's Research Institute, Melbourne, Australia 
treatment has been changed because of COVID-19. Drawing on our previous work on the ethical reasons for telling the truth to younger children (aged 5-12) we link different ethical reasons to the different types of information that could be given to children in this context. We argue that children should be given an explanation of the changes that they will directly experience, including some changes to the process of their actual medical treatment; but not about increased risk associated with these changes, unless they specifically ask for this information.

Keywords COVID-19 $\cdot$ Bioethics $\cdot$ Pre-adolescent child - Cancer care $\cdot$ Communication · Disclosure · Truthtelling

\section{Introduction}

Nathan is a nine-year-old boy who needs a bone marrow transplant (BMT) due to relapsed acute lymphoblastic leukaemia (ALL). A matched unrelated donor had been identified from an overseas registry but is no longer available due to COVID19 restrictions. Instead Nathan's mother will be the donor. This change means the transplant carries increased risks, including death. What should Nathan be told about this?

A notable feature of the COVID-19 pandemic is that children are less at risk of becoming infected or seriously unwell (Ludvigsson 2020). Despite concerns about an inflammatory syndrome associated with COVID-19, children remain "minimally affected" by the virus "overall" (Viner and Whittaker 2020, 2). Ethical discussions have consequently focused on the adult healthcare setting, with particular attention on fair allocation of critical healthcare resources such as intensive care unit (ICU) beds and ventilators. However, despite a lower risk of children becoming acutely ill with COVID-19, there nevertheless may be significant and potentially sustained effects of COVID-19 on the psychological and emotional health and well-being of children (Dalton, Rapa, and Stein 2020).

Focusing on the context of children's cancer care, we describe some of these effects and then address one specific ethical challenge that arises because of these effects. That is the question of what and how much to tell children whose cancer treatment has been changed because of COVID-19. We address this in relation to younger, primary-school aged children, drawing on our previous work on the ethical reasons for telling the truth to this age group (Hudson, Spriggs, and Gillam 2019). We have chosen to focus on children being treated for cancer, but there will be comparable impacts for children with many other serious conditions.

\section{How Children's Cancer Care is Affected by COVID-19}

In response to COVID-19, just as in past pandemics (Beaune, Nicholas, and Hocken 2012) many changes have been necessary in delivery of hospital-based cancer care to children (Balduzzi et al. 2020; Bouffet et al. 2020). Some of these changes are directed at conserving hospital resources, including theatre time and ICU beds, that might be needed for more critically ill patients. Protection of oncology healthcare providers, who are another vital and limited resource, is also an important consideration. Another key driver for changes in care delivery is to protect medically vulnerable children undergoing cancer treatment from acquiring the infection from hospital staff, visitors, and volunteers (Sullivan et al. 2020).

As a result of COVID-19, hospital staff now frequently wear personal protective equipment (PPE) while caring for children. There are significant changes in children's interactions with staff, including a reduction in activities and exchanges with allied health and psychosocial clinicians who support children's emotional well-being and development. Hospital visitors have also been significantly restricted, with often only one parent allowed to be present and no siblings or other family or friends. Children who develop a fever (a common side effect of cancer treatment) may be multiply tested for COVID-19, in case this is the cause of the fever. The COVID-19 swab procedure is uncomfortable and isolation is required while awaiting test results. For a small number of children, there may be a change in cancer treatment which will mean that the treatment they are offered is less optimal than usual standard of care. One example is allogenic Bone Marrow Transplant (BMT), where there is no matched sibling donor available. 


\section{COVID-19 and BMT}

Allogenic BMT is an intensive oncology treatment in which children with refractory or relapsed cancer undergo conditioning therapy and then receive stem cells from a donor, with the aim of curing their disease. Optimal care protocols for BMT involve a matched sibling bone marrow donor. If there is no matched donor, an anonymous matched unrelated donor (MUD) is usually sought. MUDs are identified utilizing strict protocols via international and national registries. As a result of international transportation restrictions of live tissue due to COVID-19, and concerns about the possibility of the virus being present in blood samples, attaining donations from international donors is currently extremely difficult and often not possible. This leaves the option of using a stem cell donation from a local matched unrelated donor (often none identified) or from a family member, such as a parent, who is not fully matched to the child (referred to as a haploidentical donor). Using a haploidentical donor increases the risk of complications and may lead to seriously compromised outcomes such as graft versus host disease (GVHD) or even death. Where BMT is needed urgently, however, haploidentical transplant is preferable to no transplant at all, as this would lead to certain death of the child.

\section{What Should We Tell Children About These Changes?}

Should we explicitly point out and explain to children all of the changes to their care? Should we explain what the implications are? Some of this information could be upsetting to children who are already in a frightening situation. It may seem better to spare them any further worry by acting as if it is "business as usual." After all, in most cases there is no choice about these changes, either for the children or parents. Even if there were choices or decisions to be made, these children are not mature minors and will not have any significant say about what happens. However, things are not so straightforward, ethically speaking. There are sound ethical reasons, in principle, to give honest information to children of this age about their medical treatment (Hudson, Spriggs and Gillam 2019). These reasons need careful consideration, on a case-by-case basis, before any decision is made to limit information-giving to a child.

\section{Ethical Reasons for Telling the Truth to Younger Children}

The ethical reasons to give honest information to children fall into three broad categories. One key reason for truth-telling is that, broadly speaking, it tends to promote children's well-being, both physical and psychological (Last and van Veldhuizen 1996). Children who have been given information are typically less worried, more able to cope with strange environments and happenings, and can cooperate with medical procedures, which makes the procedures go more quickly and easily for the child (Ranmal, Prictor, and Scott 2008). In practice, this suggests an ethical rule of thumb: if they will experience it, they need to know about it. This might well include knowing why things are being done, to help the child cope with unpleasant experiences by focusing on how that procedure is going to help them.

Secondly, truth-telling promotes the child's involvement in the therapeutic relationship and builds the child's trust in healthcare providers (Coyne et al. 2016; Lewis, Pantell, and Sharp 1991). Conversely, attempting to keep information hidden from a child strains the relationship and risks a major loss of trust if the child finds out about it (which is likely to happen in a hospital environment where a large number of people are interacting with the child). Over time, this involvement forms the basis for children to have input into decisions, assisting them to gradually develop the capacity for autonomous decision-making, which they will need as an adult. This also suggests that children should be told about what they are going to experience and why; preferably with the explanation coming from the healthcare providers involved, so that the child can ask questions or raise any worries that they have.

A third reason, less tangible but no less ethically important, is that truth-telling demonstrates respect for the child as a person (Coyne et al. 2016). In short, children have minds - they seek to understand themselves and the world rather than just passively experience it. This is so even when they do not have decisionmaking capacity. This reason underlines the importance of answering a child's questions honestly, whatever they are, whether or not they seem relevant or important to the healthcare provider. This needs to be done in a way that addresses the nature of the child's concern, as 
distinct from what the provider assumes the child is asking. Importantly, question-answering requires skill in speaking to the child about what they want to know and avoiding giving information which they were not seeking and did not want to know.

\section{Truth-telling about Changes in Medical Care}

In a pandemic, many changes in care will be directly experienced by children and hence need to be explained, as foreshadowed by Koller's study (Koller et al. 2010). Some things, like face masks and protective equipment, are obviously strange and may cause worry. This also applies for a child who has to be swabbed for COVID19 and isolated while waiting for the result. Without an explanation, children may develop their own explanations, that are far worse than reality (Mack and Joffe 2014): for example, the child may think "they are frightened that I am going to make them sick" or "I am having this test, that means I'm going to die of coronavirus."

\section{Telling the Truth in Relation to Change in Donor for BMT}

There are many things that we know children will experience during BMT. These include prolonged hospitalization and social isolation, treatment side effects such as nausea and vomiting, mucositis, other sources of pain and fatigue, and invasive procedures such as nasogastric tube insertion. These are no different, no matter who the donor is. As per the principles outlined above, it is important that children are told the truth about what they are likely to experience during their BMT treatment. If the donor has to be changed, as in the situation of Nathan above, from a MUD (a stranger in another country whom the child will never see or know) to the child's parent, this a change that the child will directly experience. Nathan will not be unaware of his mother preparing to be his donor, even if no-one tells him directly. He may worry what will happen to her, worries far beyond any actual risk to her. So, the ethically appropriate approach is to explain to Nathan what the change is and why, and what is involved for his mother. This approach involves sharing information with children in a way that is commensurate with their cognitive capacity and developmental stage. It also means answering honestly any questions children have- - to the level and extent that they want, in a manner that is congruent with their capacity to understand and consistent with their information preferences. This is not just passively waiting until they ask but actively creating opportunities for them to ask questions (asking them, for example, "Is there anything else you would like to know?", "Is there anything you are worried about?").

The increased risks to Nathan of receiving a BMT from a donor who is not fully matched is another matter. These risks, of GVHD or death, are not things that Nathan will experience, unless they actually occur. Nathan is not the decision-maker in this scenario; this inevitably falls to the physician and, to a lesser extent, the parent(s). Children like Nathan, therefore, do not have to weigh up the risks and benefits of changes in treatment plans. In addition, it is quite possible that describing the increased risks would increase their anxiety about a treatment decision that they are not able to influence. So, the first ethical reason for telling the truth to children, that it will in general increase their wellbeing and ability to cope with treatment, seems not apply to telling about risks. If Nathan gets GVHD, that will be the time to tell him about it, when information will help him to cope.

\section{Conclusion}

Truth-telling to seriously ill children must be assessed case by case. In general, however, young children do not really have a say in major decisions that are made about their medical treatment and this is not different, in principle, in COVID-19 times. We argue that there are not compelling reasons to tell children about increased risks and poorer prognosis that may be associated with necessary changes to treatment protocols as a result of COVID-19-unless they ask. Nevertheless, there are compelling reasons to explain changes which will be apparent to children such as changes in staff appearance, the hospital environment and visitor restrictions, and, for a child having BMT, a change from an unrelated donor to a parent donor. The principle of proactively explaining to children any changes in care which they will directly experience applies not just to the cancer context but to all contexts in which children have 
prior experience or expectations about what will happen during their care. We have focused here on changes brought about by the COVID-19 pandemic, but the principle extends to changes in care for other sorts of reasons. Openly explaining changes to children promotes their emotional well-being by giving them an understanding of what they are experiencing before they start to puzzle or worry about it.

Acknowledgements The work for this paper was supported by ARC Discovery Project DP170102906 Telling the truth to seriously ill children.

\section{References}

Balduzzi, A., E Brivio, A. Rovelli, et al. 2020. Lessons after the early management of the COVID-19 outbreak in a pediatric transplant and hemato-oncology center embedded within a COVID-19 dedicated hospital in Lombardia, Italy. Estote parati. Bone Marrow Transplant, ePub ahead of print, April 20. 10.1038/s41409-020-0895-4.

Beaune, L., D. Nicholas, and J. Hocken. 2012. Lessons learned for pediatric pandemic planning in palliative care: A case study. Journal of Palliative Care Medicine 2(5): 2-5.

Bouffet, E., J. Challinor, M. Sullivan, et al. 2020. Early advice on managing children with cancer during the COVID-19 pandemic and a call for sharing experiences. Pediatrc Blood \& Cancer, ePub ahead of print, 2 April. doi: https://doi. org/10.1002/pbc.28327.

Coyne, I., A. Amory, F. Gibson, and G. Kiernan. 2016. Information-sharing between healthcare professionals, parents and children with cancer: More than a matter of information exchange. Eurpoean Journal of Cancer Care 25(1): 141-156.

Dalton, L., E. Rapa, and A. Stein.2020. Protecting the psychological health of children through effective communication about COVID-19. The Lancet Child \& Adolescent Health 4(5): 346-347.

Hudson, N., M. Spriggs, and L. Gillam. 2019. Telling the truth to young children: Ethical reasons for information disclosure in paediatrics. Journal of Paediatrics and Child Health 55(1); 13-17.

Koller, D., D. Nicholas, R. Gearing, and O. Kalfa. 2010. Paediatric pandemic planning: Children's perspectives and recommendations. Health and Social Care in the Community 18(4): 369-377.

Last, B.F., and A.H.M. van Veldhuizen. 1996. Information about diagnosis and prognosis related to anxiety and depression in children with cancer aged 8-16 years. European Journal of Cancer 32(2): 290-294.

Lewis, C.C., R.H. Pantell, and L. Sharp. 1991. Increasing patient knowledge, satisfaction and involvement: Randomized trial of a communication intervention. Pediatrics 88(2): 351-358.

Ludvigsson, J.F. 2020. Systematic review of COVID-19 in children shows milder cases and a better prognosis than adults. Acta Paediatrica. ePub ahead of print. April 14. doi: https://doi.org/10.1111/apa.15270.

Mack, J.W., and S. Joffe. 2014. Communicating about prognosis: Ethical responsibilities of pediatricians and parents. Pediatrics 133(Supplement 1): S24-S30.

Ranmal, R., M. Prictor, and J.T. Scott. 2008. Interventions for improving communication with children and adolescents about their cancer. Cochrane Database of Systematic Reviews (4), Art. No.: CD002969.

Sullivan, M., E. Bouffet, C. Rodriguez-Galindo, et al. 2020. The COVID-19 pandemic: A rapid global response for children with cancer from SIOP, COG, SIOP-E, SIOP-PODC, IPSO, PROS, $\mathrm{CCI}$ and St Jude Global. Pediatric Blood and Cancer. ePub ahead of print, May 13. 10.22541/au.158777298.87289192.

Viner, R., and E. Whittaker. 2020. Kawasaki-like disease: Emerging complication during the COVID-19 pandemic. Lancet 395(10239): 1741-1743.

Publisher's note Springer Nature remains neutral with regard to jurisdictional claims in published maps and institutional affiliations. 